\title{
Physics in the Magnetic Configuration Space of W7-X
}

\author{
J. Geiger*, C.D. Beidler, Y. Feng, H. Maaßberg, N.B. Marushchenko, and Y. Turkin \\ Max-Planck Institut für Plasmaphysik, D-17491 Greifswald, Germany
}

(Dated: October 28, 2014)

\begin{abstract}
The neoclassical confinement and the bootstrap current are analysed in the configuration space of W7-X by self-consistent neoclassical transport simulations. Since the establishment of quasi-stationary operation is the most important goal for W7-X, the analysis concentrates on high-performance discharge scenarios in magnetic configurations which are adjusted so that bootstrap current vanishes, or, alternatively, on scenarios where the bootstrap current can be balanced by strong ECCD. Both scenarios lead to restrictions either in the configuration space or in plasma parameters and ECRH heating scenarios. Furthermore, the flexibility of the magnetic configuration space of $\mathrm{W} 7-\mathrm{X}$ is briefly described with emphasis on other physics topics of interest, for example, ballooning unstable configurations as well as configurations with a magnetic hill which might lead to interchange instability.
\end{abstract}

Accepted by PPCF, to appear online in Dec.2014, in paper Jan. 2015

\footnotetext{
*E-mail: Joachim.Geiger@ipp.mpg.de
} 


\section{INTRODUCTION}

The tokamak perspective for a reactor with stationary operation appears to be somewhat endangered, since a huge amount of recirculating power is neccessary for ECCD (electron cyclotron current drive) and/or n-NBCD (negative neutral beam current drive). Furthermore, the appearence of disruptions and the (Greenwald) density limit restrict advanced tokamak discharge scenarios. All these problems, however, do not exist in stellarators (where the plasma has much less free energy than in tokamaks). On the other hand, an optimisation of advanced stellarator configurations is mandatory: the unfavourable neoclassical transport at low collisionalities must be significantly reduced and the bootstrap current must be minimised, at least for the proper operation of an island divertor utilising low-order rationals of the rotational transform, $t$ (forming the magnetic islands within the divertor). In principle, current drive schemes can also be applied for the current control, but this goal can be obtained by the optimisation process of the magnetic configurations.

The Wendelstein 7-X stellarator (W7-X) has been optimised with respect to good MHD stability, improved neoclassical confinement, good confinement of fast particles, and low bootstrap current; for a brief overview of the optimisation process, see Refs. [1, 2] and the references therein. W7$\mathrm{X}$ belongs to the HELIAS line (HELIcal axis Advanced Stellarator) [3] where the 3D shaping of the magnetic axis allows for additional freedom for different optimisation targets. (Also the name "high-mirror advanced stellarator" was introduced for this type of magnetic configuration with good neoclassical confinement and low bootstrap current [4]). This paper concentrates on the scenario development with respect to good neoclassical confinement and low bootstrap current for quasi-stationary discharges (pulse duration up to $30 \mathrm{~min}$ ) with high performance. Establishing such discharges is the main mission of $\mathrm{W} 7-\mathrm{X}$ to demonstrate the reactor perspective of highly optimised stellarators with an island divertor [5].

It must be mentioned that open issues remain which might prevent any realistic reactor perspective of W7-X-type stellarators: i) the problem of impurity accumulation, and ii) a sufficient density control (even density profile control). For high-performance discharges, the radial electric field, $E_{r}$, evaluated from the ambipolarity condition is typically negative leading to a strong inward convection of impurities $\left(\propto Z_{I} E_{r} / T_{i}\right.$ where $Z_{I}$ is the impurity ion charge state and $T_{i}$ the ion temperature) in traditional neoclassical theory. Second, since a link exists between radial energy and particle fluxes, a corresponding link exists between power and particle sources (at least in neoclassical theory, here used in the transport simulations) which requires central refueling by pellets. 
Furthermore, a neoclassical particle transport barrier might appear outside of the power deposition zone [6] for "high-mirror advanced stellarators". This topic has not been included in the W7-X optimisation. W7-X must find solutions for both of these crucial issues.

The outcome of the W7-X optimisation process focussed on one point in the magnetic configuration space (with high mirror and an edge $t_{a}=1 ; N_{p}=5$ field periods resulting in five islands in the divertor) produced by a set of non-planar coils of five different types with identical coil currents; this "original" configuration is included in the configurational sensitivity analysis of Sec. V. The desire for experimental flexibiliy, i.e. the realisation of both low- and high-mirror configurations as well as $t_{a}=5 / 4$ and $t_{a}=5 / 6$ divertor scenarios, resulted in a new coil design (with about half the original mirror size for identical currents in the modular coils) with additional planar coils. This coil system and the flexibility of the magnetic configuration space of W7-X is briefly described in Sec. II as such a documentation is lacking to date. An equivalent configuration rather close to the "original" one can nevertheless be realised with this "new" W7-X coil system.

In this paper, quasi-stationary discharge scenarios are calculated by means of an iteration loop (up to sufficient convergence): equilibrium (by the VMEC code [7]), neoclassical transport coefficient database (by the DKES code [8]), and neoclassical transport simulation (by the NTSS code [9]); Sec. III. Here, "anomalous" transport contributions are omitted for the bulk part of the plasma with sufficiently high temperatures. The reason is simply that no reliable theory-based "anomalous" transport model yet exists for stellarators, and that the assumption of neoclassical transport in the bulk part of the plasma is supported by experimental power balance analysis; see e.g. [10-12]. Furthermore, the energy confinement time, $\tau_{\mathrm{E}}$, calculated with the assumption of neoclassical transport is in accordance [9, 13] with the ISS04 scaling [14]. Ignoring (feasible) turbulent contributions to the radial transport (it is very unlikely that such contributions affect the parallel transport), leads in all likelihood to upper limits for the energy confinement time, $\tau_{\mathrm{E}}$, and the volume averaged plasma pressure, $\langle\beta\rangle$. Decreased temperatures, e.g. due to additional turbulent transport, affect the bootstrap current densities i) through influence of the equilibrium on the magnetic configuration (reduced $\beta(r)$ ), ii) by the collisionality and radial electric field (see [15] for the mono-energetic bootstrap current coefficient), and iii) by the energy convolution based on Maxwellians. All these dependencies are rather complex, but the total bootstrap current generally decreases with reduced temperatures.

Two discharge scenarios are analysed in Sec. IV: first, "conservative" scenarios without electron cyclotron current drive (ECCD) where the magnetic configuration of $\mathrm{W} 7-\mathrm{X}$ is adjusted to min- 
imise the bootstrap current, $I_{b} \simeq 0$, and, second, scenarios with strong ECCD with $I_{b}+I_{\mathrm{ECCD}} \simeq 0$. High-performance discharges, i.e. at high density with ECRH (electron cyclotron resonance heating) in O2-mode [16], require these "conservative" scenarios since the ECCD efficiency is much too low to balance an appreciable bootstrap current. Strong ECCD is obtained only for X2-mode at intermediate or low densities, and the density, the ECCD power and the power deposition must be adjusted to achieve current balance. Since in W7-X only ECRH with up to $10 \mathrm{MW}$ at 140 $\mathrm{GHz}$ (corresponding to $B \simeq 2.5 \mathrm{~T}$ at second harmonic) is available for long pulses, neutral beam injection (NBI) scenarios are disregarded in this context (the NBI pulse duration is less than $10 \mathrm{~s}$ ). Finally, a configurational sensitivity analysis for the high-mirror configuration at $t_{a}=5 / 5$ is performed (in Sec. V) showing the impact of (small) modifications of the currents in the W7-X coil system on the neoclassical energy confinement as well as on the bootstrap current.

\section{MAGNETIC CONFIGURATION SPACE}

The large variety of W7-X configurations is obtained by the very flexible coil current system. The classification of the different magnetic configurations, however, is based on the Fourier representation $B_{m n}$ (e.g. in Boozer co-ordinates) of the magnetic field strength, $B$, on flux-surfaces,

$$
B(r, \theta, \zeta) / B_{0}=\sum_{m, n=0} b_{m n}(r) \cos \left(m \theta-n N_{p} \zeta\right)
$$

where $r$ is the flux-surface label identified with the minor radius, $\theta$ and $\zeta$ the poloidal and toroidal angle-like co-ordinates, respectively, $B_{m n}=B_{0} \cdot b_{m n}$, and $B_{0}$ is a reference magnetic field (e.g. $B_{00}$ at the outermost flux-surface).

\section{A. The coil system}

W7-X has a major radius of $R \simeq 5.5 \mathrm{~m}$, an aspect ratio of $A=R / r_{a} \simeq 10\left(r_{a}\right.$ is the plasma radius), and $N_{p}=5$ field periods. In each half field period, the coil system is composed of 5 modular and 2 planar (tilted) superconducting coils; see Fig. 1. The additional control coil (also called sweep coil) is also shown. This coil will only be used for fine-tuning of the island structure in the divertor and has nearly no impact on the magnetic configurations analysed here (and is therefore omitted in this context). The identical coils are arranged to insure stellarator symmetry in the other half of the field period. Finally, one trim coil per field period exists which is located 
at the outside of the cryostat vessel. These coils might be used for control of small error fields in the divertor (e.g. for suppressing a 1/1-island forming an envelope around the five 5/5-islands). Two poloidal cross sections of the flux surfaces are up-down symmetric: the "bean-shaped" cross section between coils of type 1 and the "triangular" one between the coils 5; see Fig. 2.

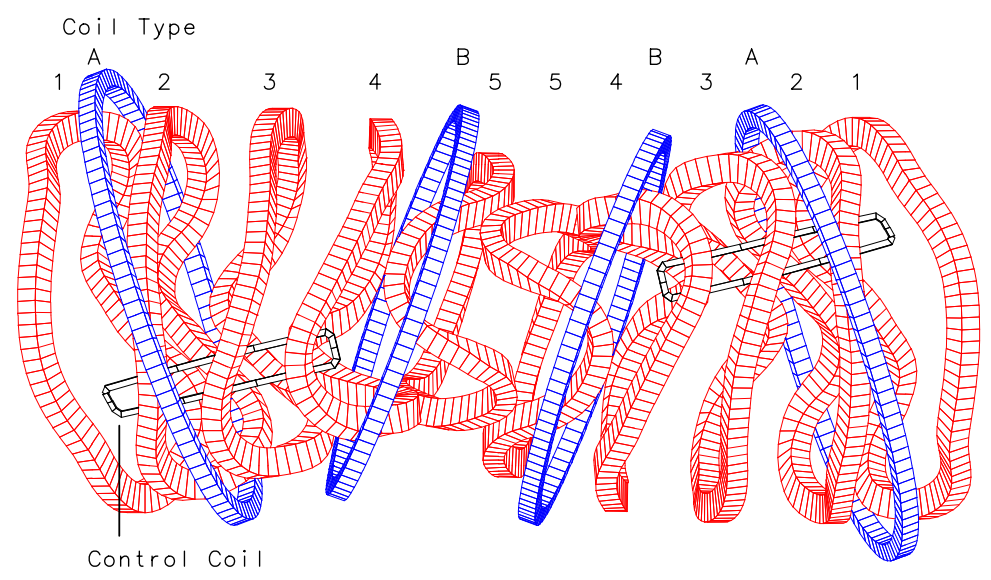

FIG. 1: (colour online) One field period of the W7-X coil system with modular (1 to 5) and planar (A and B) coil types.

All coils of a half field period have independent power supplies resulting in a great variety of magnetic configurations. The W7-X "standard" configuration is technically defined: the current ratios $i_{n}=I_{n} / I_{1}$ in all modular coils, $i_{1}$ to $i_{5}$, are identical, and there is no current in the planar coils, $i_{A}=i_{B}=0$ (see Fig. 3). For this configuration, the edge rotational transform is $t_{a}=1$ with 5 independent islands in the divertor. The toroidal mirror term, $b_{01}$, is increased if the currents in the coils close to the "bean-shaped" plane, where the local toroidal curvature (in real space) is largest, are increased compared with that in the other coils, i.e. $1>i_{2}>i_{3}>i_{4}>i_{5}$ (this might be different for special cases with shaping of the toroidal mirror form; see Sec. V). The (standard) "highmirror" configuration with $t_{a}=1$ (see Fig. 3 ) is defined by $i_{2}=0.972, i_{3}=0.926, i_{4}=0.880$, $i_{5}=0.852$, and $i_{A}=i_{B}=0$ being rather close to the original one found in the optimisation process (see Ref. [1]). An opposite ordering of the modular coil currents, $1<i_{2}<i_{3}<i_{4}<i_{5}$, leads to a reduced $b_{01}$. For $i_{A}=i_{B}>0$, the rotational transform, $t$, is reduced (the field of the planar coils has no poloidal contribution) and it is increased for $i_{A}=i_{B}<0$. For a fixed $B_{0}$, the magnetic field from the planar coils adds to the modular field and the currents in the modular coils must be reduced (and vice versa for $i_{A}=i_{B}<0$ ). $i_{A}=-i_{B}>0$ results in an inward shift of the plasma column, and $i_{A}=-i_{B}<0$ to an outward shift, but affects also the toroidal mirror term, $b_{01}$. 
One important ingredient of the optimisation was the reduction of the average toroidal curvature given by the $b_{10}$ Fourier mode in comparison to $\epsilon_{t}=r / R$. All W7-X configurations are characterised by a significantly reduced $\kappa=-b_{10} / \epsilon_{t}$ (roughly $0.3<\kappa<0.55$ ) corresponding to a very low averaged toroidal curvature. Since the magnetic field generated by the planar coils does not contribute to the $\kappa$-reduction, $\kappa$ is lowest for high $t$ and highest for low $t$ in current-free configurations, respectively; see Fig. 2.

The vacuum magnetic configuration is completely determined by the normalised coil currents, $i_{n}$. Thus, $I_{1}$ is adjusted to the desired power deposition of the ECRH (at $140 \mathrm{GHz}$ in X2- and O2mode) close to the "bean-shaped" plane; i.e. the average field in the central region, i.e. $B_{00}(0)$, is significantly lower than $2.5 \mathrm{~T}$ for high-mirror vacuum configurations.
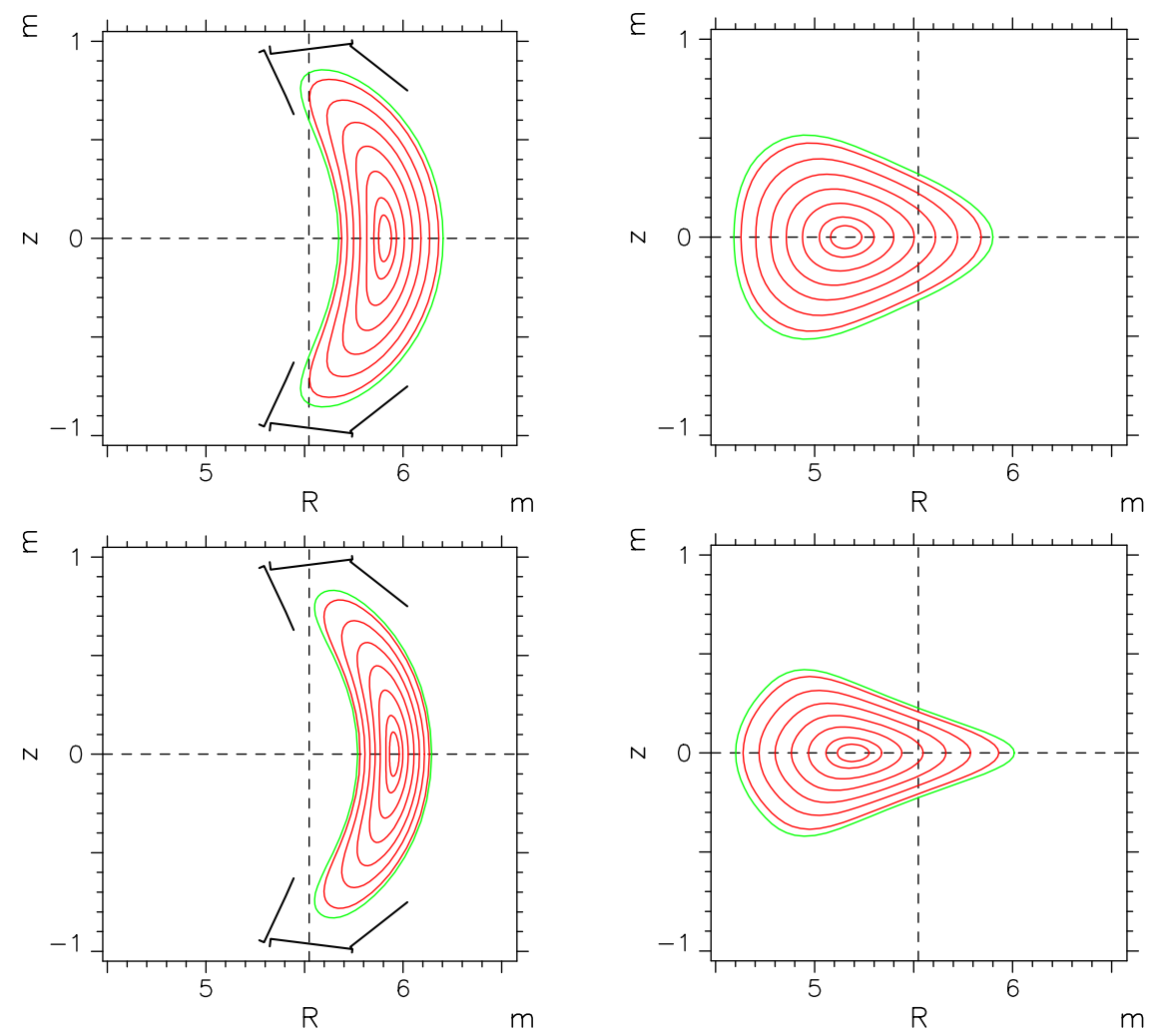

FIG. 2: (colour online) The cross section in the "bean-shaped" (left) and in the "triangular" plane (right) for the "high-mirror" configuration at low- $t\left(t_{a}=5 / 6\right.$, upper row) and at high- $t\left(t_{a}=5 / 4\right.$, lower row). The current distribution in the modular coils is identical to the "high-mirror" configuration with $t_{a}=5 / 5$, but the toroidal mirror term $b_{01}$ is reduced (increased) by about $10 \%$ (lower for low- $t$ and higher for high- $t$, respectively). 


\section{B. The main Fourier modes of W7-X configurations}

The stellarator optimisation for W7-X with the constraint of a rather narrow $b_{m n}$ Fourier spectrum "converged" to one configuration with a rather high toroidal mirror term, $b_{01} \simeq 0.1$, and with the ratio of averaged toroidal curvature to helical curvature, $b_{10} / b_{11} \approx 0.5$ at $t_{a}=1$; see $[1,17]$. The realisation of this optimisation point in the configuration space of $\mathrm{W} 7-\mathrm{X}$, however, is slightly different. Whereas $b_{10}$ is nearly identical, the $\left|b_{11}\right|$ Fourier mode with dominant impact on $t$ was slightly increased (by a modification of the modular coils) to avoid the appearance of the rational $t=5 / 6$ in the plasma core at finite $\beta$, resulting in a ratio of $b_{10} / b_{11}=0.468$ (at half the plasma radius). As a consequence, the mono-energetic radial transport coefficients at low collisionality are increased whereas the bootstrap current coefficients are reduced; see Fig. 4 in [17] for the dependence of these coefficients on $b_{10} / b_{11}$ and $b_{01}$ for a $b_{m n}$ model configuration with only these three modes. These configurations are also compared here in the sensitivity analysis of Sec. V. The calculation of all Fourier modes in magnetic co-ordinates from the coil currents is straightforward: the $B_{m n}$ as well as the Fourier representation of the flux-surfaces is performed by the VMEC code [7] and, in a second step, Boozer co-ordinates are determined by the JMC code [18]. The inverse problem of estimating the coil currents from the main $b_{m n}$-Fourier modes and $t_{a}$, however, is impossible [19]. So far, a database of precalculated magnetic configurations is used to obtain a good guess, and the final coil current distribution is estimated with an additional fine-tuning (the existing function parametrisation technique for VMEC equilibria [20] will be implemented in near future for this purpose). In fact, the back-transform from a Fourier representation with only the main $b_{m n}$ modes is not unique. For example, the toroidal mirror term, $b_{01}$, can also be slightly adjusted by an inward- or outward-shift, $i_{A}=-i_{B} \neq 0$; see Sec. V.

The main $b_{m n}$ modes of the $\mathrm{W} 7-\mathrm{X}$ configurations are the toroidal mirror term, $b_{01} \geq 0$, the averaged toroidal and helical curvatures, $b_{10}<0$ and $b_{11}<0$, respectively. In general, all other $b_{m n}$ terms are much smaller. For high-mirror configurations, the impact of $t_{a}=5 / 4,5 / 5$ and $5 / 6$ on all three $b_{m n}$ modes is rather strong: $-b_{10}$ and $-b_{11}$ decrease with $t_{a}$ whereas $b_{01}$ increases, and both $\kappa=-b_{10} R / r$ and $b_{10} / b_{11}$ decrease with $t_{a}$. For fixed $t_{a}, b_{01}$ has only a small impact on $b_{10}$ and $b_{11}$. Furthermore, the extent of the minimum of $B$ in the vicinity of the "triangular" plane can be modified by the current distribution in the modular coils, which introduces higher harmonics $b_{0 n}$ : this extent becomes broader for $b_{02}>0$ and narrower for $b_{02}<0$. The impact of this mirror form on the three main $b_{m n}$ modes, however, is fairly small. 

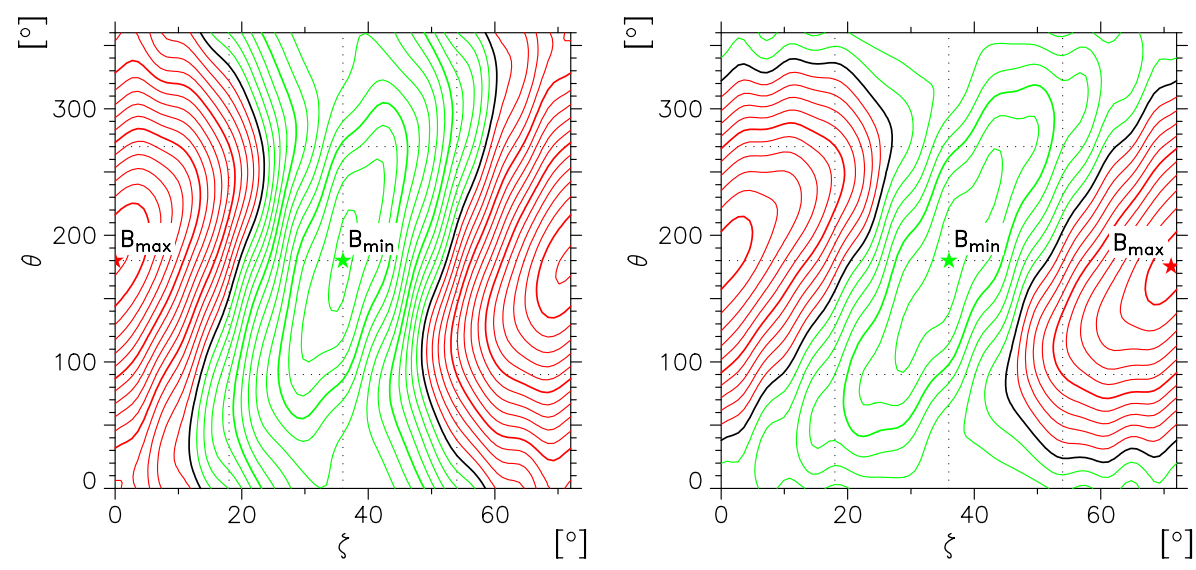

FIG. 3: (colour online) Isolines of $B$ at half the plasma radius for the "high-mirror" ( $b_{01} \simeq 0.1$; on the left) and for the "standard" configuration $\left(b_{01}=0.047\right.$; on the right $)$ at $t_{a}=1$. The "bean-shaped" plane is at $\zeta=0^{\circ}$ and the "triangular" plane at $\zeta=36^{\circ} . B=B_{00}$ is given by the black line, $B>B_{00}$ by the red and $B<B_{00}$ by the green lines; the increment $\Delta B / B_{00}$ is 0.01 .

The impact of deeply trapped particles on the neoclassical confinement properties is rather strong. For the high-mirror configurations, the confinement condition for these particles leads to the concept of quasi-isodynamic stellarators [21] with significant reduction in the radial component of their $\nabla B$-drift [22]. In a simplified approach, this drift is roughly given by the poloidal variation of $B$ in the "triangular" plane at $\zeta=36^{\circ}$; see Fig. 3, left. In these scenarios, the minimum of $B$ is quite pronounced and is located on the inner part of the flux-surfaces (i.e. at $\theta=\pi$ ). The simplified ansatz with $d B\left(\theta, \zeta=36^{\circ}\right) / d \theta=0$ only for $m=1$ and $n=-1,0,1$ leads to the same optimisation condition,

$$
b_{1-1}+b_{11}=b_{10}
$$

Consequently, the ratio $b_{1-1} / b_{11}$ must be between -0.58 and -0.50 for the configurations close to the optimised configuration. Such strong $b_{1-1}$ terms are not present in the W7-X configurations (and are neither supported by the outermost flux-surface in the optimisation nor in the realised modular coil system). In W7-X, $b_{1-1}$ is about one order of magnitude smaller than required for supressing the radial drift of deeply trapped particles (in the lowest order for only $m=1$ modes). This "missing" $b_{1-1}$ term also has an important impact on the fast-particle confinement [23], one of the W7-X optimisation criteria.

Also the "standard" configuration of Fig. 3 (on the right) has a rather pronounced minimum of $B$ at the inner side of the "triangular" plane. An equivalent approach to minimise the variation of $B$ in the helical direction with $\theta-N \zeta=0$ with only the $b_{11}, b_{10}$ and $b_{01}$ terms taken into account 
leads to the condition,

$$
b_{01}+b_{10}=0
$$

This condition, however, can only be fulfilled at a specific radius, $r$, since $b_{10}$ scales linearly with $r$ whereas $b_{01}$ is almost independent of $r$ (at least in the axis expansion of the vacuum configuration). Although the $b_{01}$ term can be adjusted in $\mathrm{W} 7-\mathrm{X}$, a good approximation to a $\sigma$-optimised configuration $[24,25]$ cannot be realised for all $r$. Nevertheless, the neoclassical confinement at low collisionalities, characterised by the "effective helical ripple", $\epsilon_{\text {eff }}$, in the $1 / \nu$-regime (for the definition, see [15] and the references therein), can be improved in comparison to the W7-X configurations with $b_{01} \simeq 0$. (A similar improvement is also obtained for the LHD "inward-shifted" configuration with $R=3.6 \mathrm{~m}$ [15] with the dominant $b_{21}$ mode locally coupling with the $b_{11}$ and $b_{31}$ modes which have different radial dependencies.)

W7-X vacuum configurations without a toroidal mirror, $b_{01} \simeq 0$, can be viewed as "classical" stellarator configurations with strong optimisation due to small averaged toroidal curvature, $\kappa$. This becomes obvious from the estimation of the "effective helical ripple" where

$$
\epsilon_{\mathrm{eff}} \approx-b_{11} \kappa^{4 / 3}
$$

is found with all $b_{m n}$ modes included for the calculation of $\epsilon_{\text {eff }}$. With finite $\beta$, however, the $b_{01}$ mirror term becomes negative leading to a fairly pronounced minimum of $B$ at the outer side of the "bean-shaped" plane (i.e. at $\theta=0$ ) where the (local) toroidal curvature dominates. A significant fraction of trapped particles in the vicinity of this minimum can exist dominating the radial transport and resulting in a strong degradation of the neoclassical confinement. Such configurations with an inverted mirror term, $b_{01}<0$, are of additional interest for investigating the roles of ballooning instability and trapped electron modes [26].

In a quite different region of the W7-X configuration space at low- $t_{a}$ and very high $b_{01}>0.2$, a vacuum magnetic hill appears [27]. These configurations are predicted to be Mercier unstable similarly to the inward-shifted LHD configurations $[14,28]$. This analysis of the impact on confinement can also be performed in W7-X and compared to the LHD findings. High interest in such experimental investigations exists to assess the importance of a magnetic well which suppresses interchange activity at low $\beta$.

The impact of pressure, i.e. the $\beta$ profile, is significant only for the $b_{00}$ and the $b_{01}$ Fourier modes which are reduced with rising $\beta$. In general, $t$ is only weakly affected (depending on the $\beta$-profile as well as on the configuration), as both the bootstrap current and the ECCD affect the rotational 
transform more strongly. The impact on $b_{10}$ and $b_{11}$ is rather small (both terms become slightly less negative). Both $\kappa=-b_{10} / \epsilon_{t}$ and $b_{10} / b_{11}$ weakly decrease with $\langle\beta\rangle$. The reduction of the toroidal mirror term for high $b_{01}$ leads to a significant improvement of neoclassical confinement. For W7-X, $\epsilon_{\mathrm{eff}}$ decreases with at least moderate $\langle\beta\rangle$ which is a quite different behavior to that of LHD configurations [29] with strong Shafranov shift [30].

Finally, the radial dependence of $b_{10} / b_{11}$ has an important consequence for the bootstrap current density profile (see Sec. IV A). In agreement with the axis-expansion of the Fourier modes, $b_{m n} \propto r^{m}, b_{10} / b_{11}$ is nearly constant for the inner radii, but increases at the outer radii. Following the analysis of Fig. 4 in Ref. [17], the bootstrap current coefficients become negative at small $r$ and positive at outer $r$ for an adjusted $b_{01}$-mirror term. In addition to the thermodynamic forces for neoclassical transport (i.e. density and temperature gradients as well as the radial electric field), this feature can lead to negative bootstrap current densities at the inner radii and to positive ones at the outer radii. For such a scenario, the shear, $t^{\prime}$, may be increased.

\section{SELF-CONSISTENT TRANSPORT SIMULATIONS}

The neoclassical mono-energetic transport coefficients are calculated with the DKES code [8] and depend only on the $b_{m n}$-spectrum for each radius and $t(r)$. In particular, the bootstrap current coefficient is strongly affected by $t$, e.g. in the ECCD scenarios (roughly scaling with $1 / t$ at low collisionalities; see Sec. IV B). The full thermal neoclassical transport matrix is obtained by energy convolution of the three mono-energetic transport coefficients stored in a database for each configuration as a function of radius, collisionality and radial electric field. In the energy convolution, parallel momentum corrections [31] are included to both the bootstrap current densities and the parallel conductivity (collision operator used by DKES does not conserve parallel momentum). These neoclassical coefficients are used in the transport simulations by the NTSS code [9] to calculate the radial particle and energy fluxes, the bootstrap current densities as well as the transient current diffusion (both on the skin-time of a few seconds and on the $L / R$-time which is typically one order of magnitude larger). The radial electric field entering both in the transport coefficients and in the thermodynamic forces is calculated from the ambipolarity condition of the radial particle fluxes by solving a diffusion equation (in this way, a unique $E_{r}$ is obtained; see [9] for more details). Only at the outermost radii, with low temperature, is an additional "anomalous" transport assumed. With the energy fluxes for all species, the energy balance is solved and the temperature 
profiles are calculated.

The ECRH power deposition and the ECCD are calculated with the TRAVIS ray-tracing code [32] which is coupled to NTSS. In TRAVIS, the ECCD can be calculated in the collisional and collisionless limits (with parallel momentum conservation), or finite collisionality effects [33] may be included. NBI deposition as well as NBCD are included in a simplified model assuming slowingdown on flux-surfaces. In the transport simulations, $Z_{\text {eff }}=1.5$ (with $C^{6+}$ ) independent of $r$ is assumed, however, the impurity flux is taken into account for evaluating $E_{r}$. From $Z_{\text {eff }}$, a rough estimate of radiative losses is obtained and included in the energy balance.

So far, the particle balance is disregarded for calculating the density profile; a fixed $n_{e}(r)$ is simply assumed. In the simulations presented here, the shape of the density profile is identical, and only its central value, $n_{e}(0)$, is varied. The reason for this approach is not related to the particle fluxes (they are used for estimating $E_{r}$ ), but to the particle sources. Particle sources at outer radii are gas puffing (which can be calculated by the EIRENE code [34]) and recycling (mainly from the island divertor targets). In order to calculate the recycling sources, a coupling with the edge transport modelling, i.e. the EMC3/EIRENE code $[35,36]$, is necessary, but really not at all trivial (e.g. the common boundary conditions at the plasma edge). For central power deposition, also central particle sources are needed [6], which might lead to density control problems at outer radii. Without these central particle sources, the density profiles are predicted to become very hollow as has been observed in LHD [12,37]. For such a scenario, pellet injection might be mandatory for controlling the full density profile. (In the near future, a stellarator version of the HPI2 pellet ablation code [38] will be used for the transport simulations). With these restrictions, the transport simulations are not completely self-consistent.

The simulated $\beta$ and current density profiles (bootstrap, ECCD and, in principle, NBCD) are the input for the VMEC equilibrium code [7], the results of which are expressed in a Boozer coordinate representation using the JMC code [18]. The new $b_{m n}$-spectrum and $t$-profile are used by DKES for the next iteration step until sufficient convergence is reached (e.g. total current of a few kA is tolerable for island divertor operation).

In Sec. IV, only two discharge scenarios are described. In a "conservative" scheme, the total bootstrap current, $I_{b}$, is minimised by a proper adjustment of only the toroidal mirror term, $b_{01}$, since the ECCD efficiency is very low at high densities (ECRH in O2-mode), required in quasistationary discharges with high performance, i.e. high $\langle\beta\rangle$; see Sec. IV A. In the second scenario at lower $b_{01}$ (Sec. IV B), e.g. in the "standard" configuration, the bootstrap current is balanced by 
strong counter-ECCD significantly affecting the $t$-profile. This scenario leads to constraints on the density and the ECRH power: $n_{e}$ must be much lower (ECRH in X2-mode) and the ECCD power has to be increased. Here, the "free" parameters in the iteration for vanishing total current are the density, the ECRH power and (partly) the ECCD deposition (i.e. all coil current ratios, $i_{n}$, are fixed). Here, more steps are needed in the iteration since the bootstrap current coefficients depend sensitively on the $t$-profile as well as the balance of ECCD and $I_{b}$ on the density and heating power.

\section{CURRENT-FREE DISCHARGE SCENARIOS}

For quasi-stationary operation of $\mathrm{W} 7-\mathrm{X}$ with a proper functioning of the island divertor, total current contol is needed, i.e. the bootstrap current must vanish, $I_{b} \simeq 0$, or it must be balanced by counter-ECCD, $I_{b}+I_{\mathrm{ECCD}} \simeq 0$. For the first scenario, the magnetic configuration must be adjusted, i.e. mainly the $b_{01}$ term, which is one criterion of the W7-X optimisation. The second scenario allows for much more freedom with respect to the magnetic configurations of interest.

\section{A. Conservative Scenarios without ECCD}

Starting point for these "conservative" scenarios is the selection of the central density for the final phase, the ECRH heating power and the island configuration, i.e. $t_{a}$. High performance at high $\langle\beta\rangle$ requires densities above the X2 cut-off $\left(\simeq 1.2 \cdot 10^{20} \mathrm{~m}^{-3}\right)$. Here, an O2-scenario with $n_{e}(0)=1.5 \cdot 10^{20} \mathrm{~m}^{-3}$ at $5 \mathrm{MW}$ ECRH power with peaked deposition close to the magnetic axis is choosen as final target of the iteration process. In each iteration step, the equilibrium calculation with the $\beta$-profile and the current density profile from the previous iteration step included are used for updating the DKES-database and for the new transport simulation. The iteration is assumed to be converged if nearly identical $I_{b} \simeq 0, \tau_{\mathrm{E}}$ and $\langle\beta\rangle$ (and profiles) with respect to the previous step are obtained. For $t_{a}=5 / 5$, the toroidal mirror term for $I_{b} \simeq 0$ is $b_{01}=0.108$ in vacuum and slightly reduced for the converged configuration at $\langle\beta\rangle=3.06 \%(\beta(0) \simeq 7 \%)$; see Tab. I. The finite $\beta(r)$ reduces $\epsilon_{\text {eff }}$ in the main confinement region (although $\epsilon_{\text {eff }}$ increases further toward the edge). The total bootstrap current is sufficiently small $(\simeq 3.4 \mathrm{kA})$ as the ion $(\simeq 13 \mathrm{kA})$ and electron $(\simeq-10 \mathrm{kA})$ contributions nearly cancel each other; see Fig. 4 (lower left plot). The bootstrap current density, $j_{b}$, is negative at the inner radii and positive at the outer radii resulting in a small reduction of the inner $t$ values. The energy confinement time, $\tau_{\mathrm{E}}$, significantly exceeds the 
one from the ISS04 scaling [14] (the normalised $\tau_{\mathrm{E}}^{\mathrm{n}}=\tau_{\mathrm{E}} / \tau_{\mathrm{E}}^{\mathrm{ISS} 04}$ is also given in Tab. I to eliminate the volume dependence).
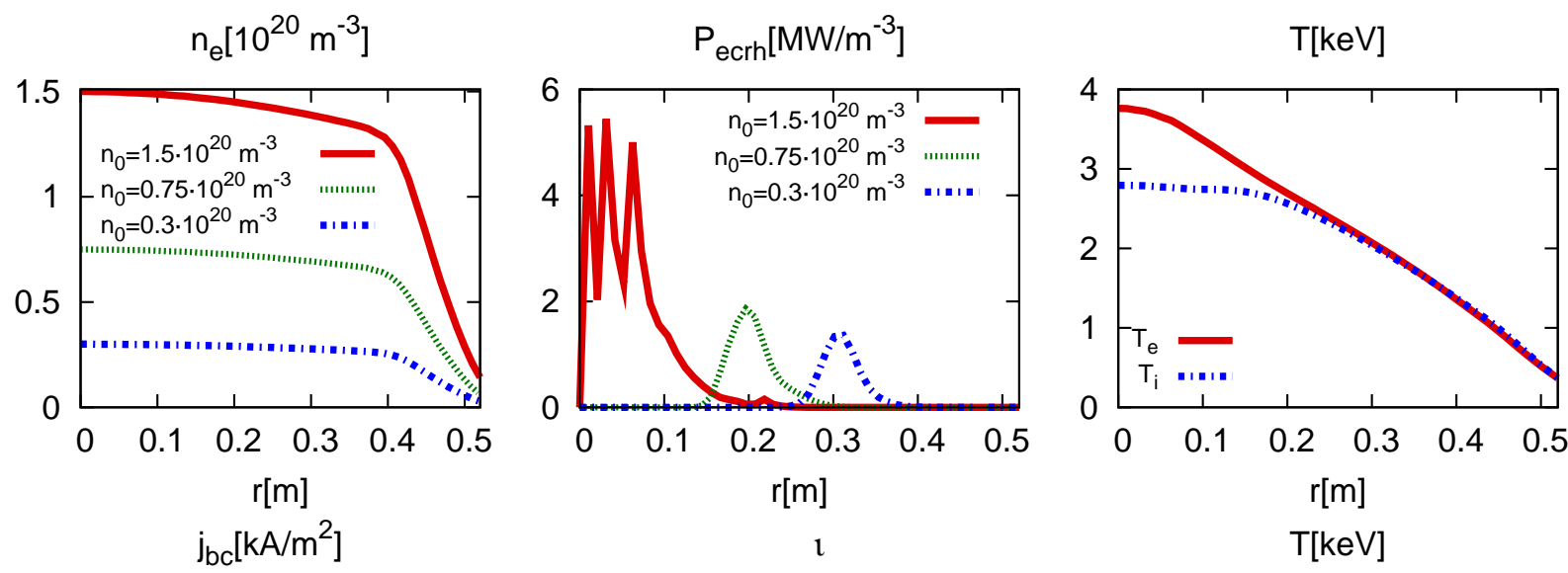

1

$\mathrm{T}[\mathrm{keV}]$
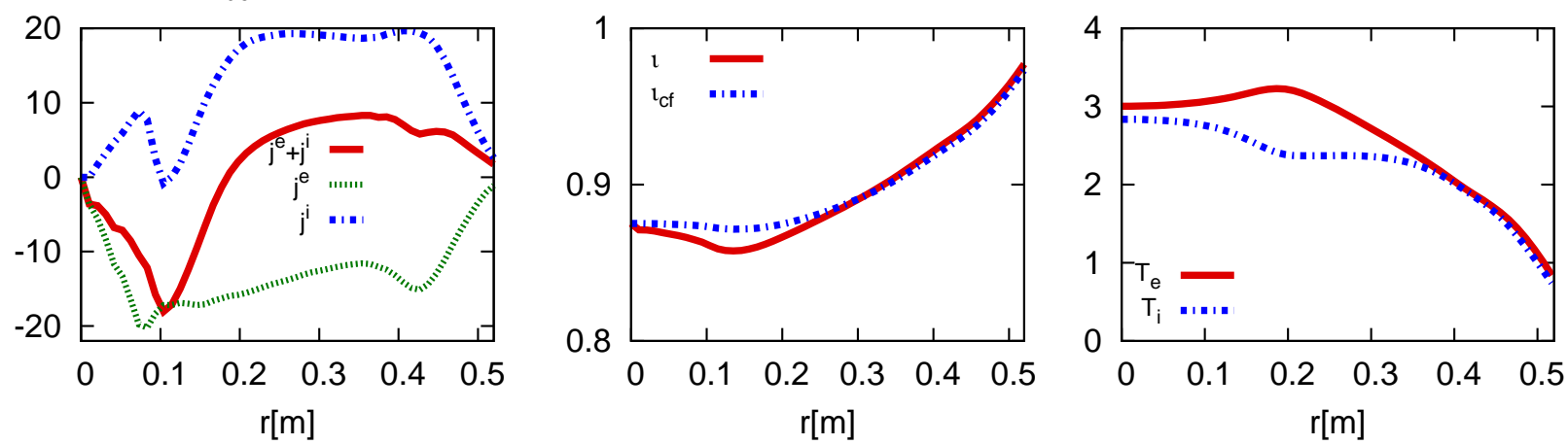

FIG. 4: (colour online) Upper line: density profiles (left), the ECRH power deposition profiles (for all scenarios; center), the $T_{e}$ and $T_{i}$ profiles for the $\mathrm{O} 2$ case (right). Lower line: the bootstrap current density profiles for the $\mathrm{O} 2$ case (left), the $t$-profile (where $t_{c f}$ is the current-free one), and the $T_{e}$ and $T_{i}$ profiles for the $\mathrm{X} 2$ case with $n_{e}(0)=0.75 \cdot 10^{20} \mathrm{~m}^{-3}$ (right).

In this "conservative" scheme, all coil currents and launching angles of the different EC beams are identical in the early phase of the discharge. For the very early phase, a value of $n_{e}(0)=0.3 \cdot 10^{20}$ $\mathrm{m}^{-3}$ was chosen also with $5 \mathrm{MW}$ ECRH in X2-mode. For this case, only the vacuum configuration was used (no iteration). For the $\langle\beta\rangle \simeq 0.7 \%$ found in this simulation, the small change in the $b_{m n}$ spectrum can be ignored. The ECRH position for this low- $\beta$ case is strongly off-axis on the lowfield side to allow for central deposition in the high- $\beta$ phase in O2-mode. For this low-density case, $\tau_{\mathrm{E}}$ exceeds $\tau_{\mathrm{E}}^{\mathrm{ISS} 04}$ only slightly due to the strong off-axis ECRH deposition. Also for this low-density case, the bootstrap current is found to be sufficiently small.

Next, the density is increased to $n_{e}(0)=0.75 \cdot 10^{20} \mathrm{~m}^{-3}$ in preparation for the transition from $\mathrm{X} 2$ to $\mathrm{O} 2$-mode by switching the polarisation of the EC beams (simulation again with $5 \mathrm{MW}$ power). 


\begin{tabular}{c|c||c|cc|c||c|ccccc}
\hline$t_{a}$ & $\begin{array}{c}n_{e}(0) \\
{\left[10^{20} \mathrm{~m}^{-3}\right]}\end{array}$ & $\begin{array}{c}I_{b} \\
{[\mathrm{kA}]}\end{array}$ & $\begin{array}{c}\tau_{\mathrm{E}} \\
{[\mathrm{s}]}\end{array}$ & $\tau_{\mathrm{E}}^{\mathrm{n}}$ & $\begin{array}{c}\langle\beta\rangle \\
{[\%]}\end{array}$ & $\begin{array}{c}\text { Vol. } \\
{\left[\mathrm{m}^{3}\right]}\end{array}$ & $\begin{array}{c}B_{00}(0) \\
{[\mathrm{T}]}\end{array}$ & $b_{01}(0)$ & $\epsilon_{\mathrm{eff}}$ & $\kappa$ & $b_{10} / b_{11}$ \\
{$[\%]$} & & \\
\hline \hline \multirow{2}{*}{$\frac{5}{5}$} & 0.30 & 6.0 & 0.147 & 1.32 & 0.75 & 29.4 & 2.38 & 0.108 & 2.64 & 0.450 & 0.474 \\
& 0.75 & 5.0 & 0.351 & 1.98 & 1.84 & 29.3 & 2.32 & 0.100 & 2.41 & 0.447 & 0.479 \\
& 1.50 & 3.4 & 0.605 & 2.33 & 3.06 & 29.7 & 2.27 & 0.094 & 2.16 & 0.440 & 0.480 \\
\hline \multirow{5}{*}{$\frac{5}{6}$} & 0.30 & 5.0 & 0.126 & 1.36 & 0.91 & 28.5 & 2.12 & 0.244 & 6.25 & 0.577 & 0.608 \\
& 0.75 & -1.6 & 0.260 & 1.77 & 1.92 & 28.5 & 2.06 & 0.236 & 5.71 & 0.586 & 0.602 \\
& 1.50 & 2.1 & 0.463 & 2.10 & 3.15 & 28.4 & 2.08 & 0.230 & 5.10 & 0.586 & 0.614 \\
\hline \multirow{5}{*}{$\frac{5}{4}$} & 0.30 & 6.0 & 0.125 & 1.32 & 0.70 & 23.8 & 2.51 & 0.040 & 0.89 & 0.353 & 0.388 \\
& 0.75 & 8.6 & 0.370 & 2.42 & 2.11 & 23.8 & 2.46 & 0.037 & 0.87 & 0.347 & 0.385 \\
& 1.50 & 0.2 & 0.596 & 2.70 & 3.32 & 23.9 & 2.39 & 0.027 & 0.70 & 0.331 & 0.375 \\
\hline
\end{tabular}

TABLE I: Conservative scenarios without ECCD for $t_{a}=5 / 5,5 / 6$ and $5 / 4$. On-axis ECRH deposition in O2-mode is modelled only for the high density cases; the coil currents in each $t_{a}$ scenario are kept fixed for the different $n_{e}(0)$. The configuration parameters, $\epsilon_{\mathrm{eff}}, \kappa$ and $b_{10} / b_{11}$, are given at half the plasma radius. The transport simulation results are obtained for 5 MW ECRH for both X2- and O2-mode.

This transition is analysed in detail in Ref. [16] for the "standard" configuration. With increased $\beta$ at this density, the ECRH deposition is shifted inward compared to the vacuum scenario. Here, an iteration of the $\beta$-profile is performed; for the temperture profiles, see Fig. 4 (lower right plot). Again, the impact of $j_{b}(r)$ on the $t$-profile is small. For all these cases with increased $n_{e}(0)$ and the transition from X2- to O2-mode by (fast) changing of the beam polarisation, the bootstrap current remains small enough and no ECCD is needed to control the island configuration in the divertor. For both $t_{a}=5 / 4$ and $t_{a}=5 / 6$, the same scenarios as for $t_{a}=5 / 5$ are analysed. The high- $t$ configurations have both a smaller $\kappa=-b_{10} R / r$ and $b_{10} / b_{11}$ ratio, and a smaller $b_{01}$ mirror term should be sufficient for $I_{b} \simeq 0$. For the $n_{e}(0)=1.5 \cdot 10^{20} \mathrm{~m}^{-3}$, O2-mode scenario, a vacuum value of only $b_{01}=0.04$ (and further reduced with finite $\beta$ ) is needed for $I_{b} \simeq 0$. For this rather low toroidal mirror term, $\epsilon_{\text {eff }}$ is much lower in the main confinement region compared to the other $t_{a}$ scenarios allowing for much higher $T_{e}(0)$ although the plasma volume is reduced. The electron and ion contributions to $I_{b}$ cancel each other globally, but not locally. In the central region, $j_{b}<0$ (the electron contribution dominates) resulting in $t<1$. The electron contribution to $I_{b}$ dominates at low density whereas the ion contribution predominates at the intermediate density; the impact 
on $t(r)$ is small in both cases.

Finally, results for the low- $t$ scenario with $t_{a}=5 / 6$ are described. For the high-performance target with $n_{e}(0)=1.5 \cdot 10^{20} \mathrm{~m}^{-3}$ with O2-mode, a very large $b_{01} \simeq 0.25$ term in vacuum is required for $I_{b} \simeq 0$. The low- $t$ configurations have both the largest $\kappa$ and $b_{10} / b_{11}$ in comparison with the higher- $t_{a}$ ones. For this configuration, $\epsilon_{\mathrm{eff}} \geq 5 \%$ in the main confinement region is rather high. The rather large $\langle\beta\rangle$ is attributed to the significantly reduced $B_{00}$ for $B \simeq 2.5 \mathrm{~T}$ at the ECRH deposition in the "bean-shaped" plane. Also for these scenarios, the simulations show only a moderate impact of $j_{b}$ on the $t$ profile.

\section{B. Scenarios with strong counter-ECCD}

Here, the "standard" configuration with identical modular currents, $I_{n}$, and $I_{A}=I_{B}=0$ for $t_{a}=5 / 5$ (see. Sec. II A) is selected for scenarios with ECCD compensation of the bootstrap current, $I_{b}+I_{\mathrm{ECCD}} \simeq 0$. ECCD in O2-mode is not efficient enough by far, but the ECCD efficiency is also not sufficient at higher density $\left(n_{e}(0) \simeq 10^{20} \mathrm{~m}^{-3}\right)$ and for moderate power levels in X2mode. Consequently, $n_{e}(0)$, the heating power, as well as the power deposition region are "free" parameters in the iteration process, whereas $j(r)=j_{b}+j_{\mathrm{ECCD}}$ (with a dominant impact of the EC driven current density, $\left.j_{\mathrm{ECCD}}\right)$ and $\beta(r)$ determine the plasma response of the magnetic configuration. In these iterations, counter-ECCD significantly reduces $t$ at the ECCD position resulting in a strong increase of the bootstrap current coefficients (roughly scaling as $1 / t$ at low collisionalities). Consequently, more iteration steps are needed for scenarios with strong ECCD compared to those without ECCD (Sec. IV A).

Within the iteration the central density has to be reduced, $n_{e}(0)=0.67 \cdot 10^{20} \mathrm{~m}^{-3}$ (for the profile shape, see Fig. 4; upper left plot), and the ECRH power increased to $7 \mathrm{MW}$ with a deposition at around one third of the plasma radius. Only for this rather strong off-axis deposition could the $t$-reduction be limited to a minimum value of 0.45 (the change in $t(r)$ due to the local current scales with $\left.\Delta t \propto 1 / r^{2}\right)$. The converged profiles are given in Fig. 5. The total bootstrap current is $I_{b}=82.1 \mathrm{kA}$ (with the main contribution from electrons), and $I_{\mathrm{ECCD}}=-81.0 \mathrm{kA}$ is found; the rather small difference is negligible for the island divertor operation. Even for this rather low $n_{e},\langle\beta\rangle=2.2 \%$ (with $\beta(0) \simeq 4 \%$ ) is fairly high due to the good neoclassical confinement with $\epsilon_{\mathrm{eff}} \leq 0.6 \%$ in the main confinement region. The energy confinement time is $\tau_{\mathrm{E}}=0.34 \mathrm{~s}$ (and normalised $\tau_{\mathrm{E}}^{\mathrm{n}}=2.7$ ). For higher density or lower ECRH power, no current balance $I_{b}+I_{\mathrm{ECCD}} \simeq 0$ 

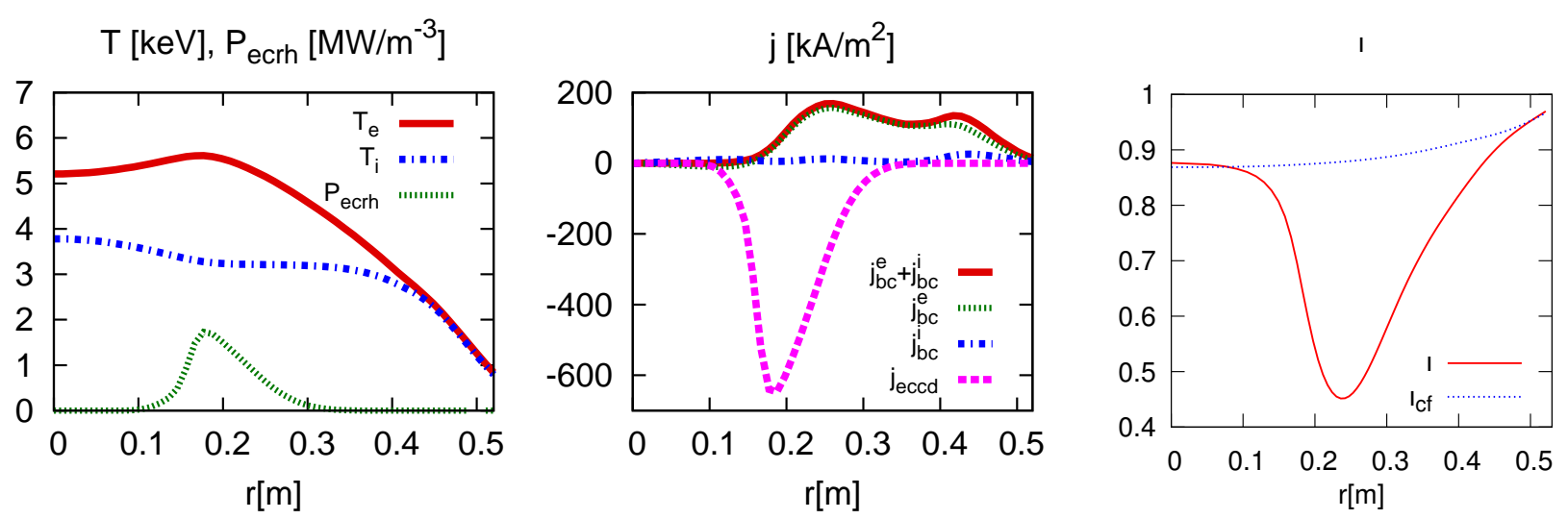

FIG. 5: (colour online) $T_{e}$ and $T_{i}$ profiles (power deposition is indicated), left, the current density profiles, $j_{\mathrm{ECCD}}$ and $j_{b}$ (center), and the $t$-profile (right).

could be obtained in this fairly strong off-axis deposition scenario $\left(I_{\mathrm{ECCD}} \propto P_{\mathrm{ECCD}} / n_{e}\right.$ behaves quite differently to the $I_{b}$ dependence on power and density; see Ref. [16]).

The shape of $t(r)$ is characterised by negative shear $t^{\prime}<0$ inside the ECCD position and positive shear $t^{\prime}>0$ outside. (In the tokamak community, the sign convention is the opposite: $t^{\prime}<0$ is designated as "positive" shear.) In W7-AS, strong mode activity was found mainly in the $t^{\prime}<0$ region for co-ECCD, but with coupling to other low-order $t$-rationals with $t^{\prime}>0$ [39]. In equivalent discharges with strong counter-ECCD, i.e. with only strong positive shear, however, no mode activity could be observed. For the counter-ECCD scenario of Fig. 5 with $t=1 / 2$ within the $t$-profile, island formation might be expected at this low-order rational.

The situation becomes even more sensitive because of the $t$-dependence of the bootstrap current and of the neoclassical confinement for an equivalent simulation with on-axis counter-ECCD. The ECCD current densities close to the axis become formally very large leading to strongly negative $t$. Consequently, the role of the $t \simeq 0$ region on the confinement becomes essential, but there are two further issues. The first one is related to ergodisation of the magnetic field, i.e. the assumption of nested flux-surfaces (in the VMEC code) is violated without rotational transform (equivalent to the start-up of tokamak discharges). Then, neither particle and energy confinement nor localised current densities can be expected. The second issue is a neoclassical one: particle and energy confinement are determined by the plateau transport, which scales with $1 / t$ and extends to very low collisionalities [15] (confirmed in the DKES calculations); also the bootstrap current coefficients scale with $1 / t$ at low $\nu^{*}$. These neoclassical scalings reflect the strong deviation of particle orbits from flux-surfaces (even if they exist). Because of these issues at very low (but finite) $t$, the 
assumption of good confinement is questionable. Counter-ECCD discharges in W7-AS showed completely flat density and temperature profiles in the central region consistent with $t \simeq 0$ in the current balance simulations [39].

The transport simulations for W7-X counter-ECCD reflect these findings. Very large transport coefficients are assumed within the central region with e.g. $t<0.05$, and only the neoclassical ones outside (i.e. with DKES data for $t \geq 0.05$ ). With the large neoclassical transport coefficients at low $t$, the temperature gradients (and also $E_{r}$ ) are strongly decreased resulting in strongly reduced thermodynamic forces for driving the bootstrap current density in this region. Such a transport and current balance simulation is performed (also for the W7-X "standard" configuration) with $n_{e}(0)=0.5 \cdot 10^{20} \mathrm{~m}^{-3}$ at $5 \mathrm{MW}$ heating power in X2-mode with optimum launch angles for maximum central ECCD. In the central region $r<0.21 \mathrm{~m}$ with $t<0.05$, the ECCD current density profile is assumed to be completely flat (and $j_{b}=0$ ) allowing for $t \simeq 0$. A total ECCD current of $I_{\mathrm{ECCD}}=-72.4 \mathrm{kA}$ is obtained, balancing the bootstrap current of roughly $I_{b}=67.7 \mathrm{kA}$ (with dominant contribution by electrons $) ;\langle\beta\rangle=1.6 \%, \tau_{\mathrm{E}}=0.325 \mathrm{~s}\left(\tau_{\mathrm{E}}^{\mathrm{n}}=2.4\right)$, and $\epsilon_{\mathrm{eff}}>0.6 \%$ in the main confinement region is found for the converged iteration.

The clear disadvantage of these strong counter-ECCD scenarios is the significantly reduced performance since they are resticted to fairly low densities; no access to high densities with ECCD in O2-mode exists. Furthermore, these scenarios are very sensitive to the current density as well as the density and temperature profiles. Consequently, such scenarios should be investigated in W7$\mathrm{X}$ discharges, but the "conservative" schemes of Sec. IV A without strong ECCD will be more attractive for quasi-stationary operation with edge $t_{a}$-control for the island divertor.

\section{CONFIGURATIONAL SENSITIVITY ANALYSIS}

The dependence of both the neoclassical confinement and the bootstrap current on the magnetic configuration is very complex. In general, the target function as well as the specific weights for both topics within the optimisation process might force a compromise with respect to high $\tau_{\mathrm{E}}$, high $\langle\beta\rangle$ and low $I_{b}$. As is shown in Sec. IV, the weight for a minimised $I_{b}$ must be significantly increased compared to the "original" W7-X optimisation under the constraint of a proper functioning of the island divertor. In this section, the configurational sensitivity of $\tau_{\mathrm{E}},\langle\beta\rangle$ and $I_{b}$ is analysed with the (standard) "high-mirror" configuration being the reference case A in Tab. II; the coil current ratios, $i_{n}$, are given in Sec. II A. For all configurations, a fixed parabolic $\beta$-profile with 
$\langle\beta\rangle=2 \%$ is assumed, and an iteration for an equilibrium consistent with the transport simulations is omitted.

For the transport simulations, $n_{e}(0)=0.75 \cdot 10^{20} \mathrm{~m}^{-3}$ with the profile shape of Fig. 4 (upper left plot) with $5 \mathrm{MW}$ ECRH (without ECCD) and both on-axis and off-axis deposition are choosen. Except for the case of $b_{01}$ variation in configurations $\mathbf{B}$ and $\mathbf{C}$, the toroidal mirror term is $b_{01} \simeq 0.1$ in vacuum and slightly lower at finite $\beta$. With central power deposition at this rather low density, a pronounced "electron-root" feature (up to about $30 \%$ of the plasma radius) is obtained for all the "high-mirror" configurations in Tab. II. This neoclassical "electron-root" with strongly positive $E_{r}$ and significantly improved electron heat confinement has been experimentally observed in numerous stellarators consistent with the theoretical predictions [10]. This "electron-root" feature leads to an additional contribution of between $25 \%$ and $70 \%$ to the bootstrap current, $I_{b}$. For comparison, a second set of transport simulations is performed for off-axis power deposition (at $r \sim 0.18 \mathrm{~m}$ ) without this "electron-root" feature.

For the (standard) "high-mirror" configuration (case A) with on-axis ECRH deposition, $I_{b}$ is

\begin{tabular}{c||c|cc|c||c|cc|c||c|ccc}
\multicolumn{10}{c}{ on-axis ECRH } & \multicolumn{10}{c}{ off-axis ECRH } \\
\hline config. & $\begin{array}{c}I_{b} \\
{[\mathrm{kA}]}\end{array}$ & $\begin{array}{c}\tau_{\mathrm{E}} \\
{[\mathrm{s}]}\end{array}$ & $\tau_{\mathrm{E}}^{\mathrm{n}}$ & $\begin{array}{c}\langle\beta\rangle \\
{[\%]}\end{array}$ & $\begin{array}{c}I_{b} \\
{[\mathrm{kA}]}\end{array}$ & $\begin{array}{c}\tau_{\mathrm{E}} \\
{[\mathrm{s}]}\end{array}$ & $\begin{array}{c}\tau_{\mathrm{E}}^{\mathrm{n}} \\
\mathbf{A}\end{array}$ & $\begin{array}{c}\langle\beta\rangle \\
{[\%]}\end{array}$ & $\begin{array}{c}\text { Vol. } \\
{\left[\mathrm{m}^{3}\right]}\end{array}$ & $\begin{array}{c}\epsilon_{\mathrm{eff}} \\
{[\%]}\end{array}$ & $\kappa$ & $b_{10} / b_{11}$ \\
\hline \hline $\mathbf{A}$ & 18.4 & 0.386 & 2.23 & 2.10 & 10.6 & 0.376 & 2.12 & 1.95 & 29.4 & 2.04 & 0.441 & 0.473 \\
\hline $\mathbf{B}$ & 28.9 & 0.420 & 2.39 & 2.17 & 19.7 & 0.395 & 2.21 & 1.95 & 29.4 & 1.40 & 0.429 & 0.461 \\
$\mathbf{C}$ & 10.5 & 0.330 & 2.11 & 2.04 & 2.1 & 0.310 & 1.95 & 1.83 & 27.1 & 2.74 & 0.452 & 0.484 \\
\hline $\mathbf{D}$ & 17.1 & 0.352 & 2.29 & 2.12 & 9.5 & 0.342 & 2.18 & 1.96 & 26.6 & 2.04 & 0.440 & 0.471 \\
$\mathbf{E}$ & 19.2 & 0.352 & 2.33 & 2.16 & 11.2 & 0.342 & 2.22 & 2.00 & 26.2 & 1.97 & 0.445 & 0.480 \\
\hline $\mathbf{F}$ & 8.0 & 0.358 & 1.99 & 1.87 & 3.1 & 0.349 & 1.91 & 1.74 & 29.5 & 2.45 & 0.438 & 0.475 \\
$\mathbf{G}$ & 25.9 & 0.390 & 2.35 & 2.22 & 15.5 & 0.378 & 2.24 & 2.05 & 29.2 & 1.72 & 0.444 & 0.472 \\
\hline \hline H & 40.7 & 0.398 & 2.30 & 2.16 & 32.0 & 0.388 & 2.19 & 2.01 & 29.4 & 1.98 & 0.441 & 0.473 \\
\hline $\mathbf{I}$ & 44.4 & 0.427 & 2.81 & 2.53 & 29.0 & 0.416 & 2.67 & 2.35 & 27.4 & 1.15 & 0.457 & 0.510 \\
\hline
\end{tabular}

TABLE II: Impact of small configuration modifications on plasma performance compared to the (standard) "high-mirror" configuration (reference configuration A); the definition of the different configurations is described in the text. The configuration parameters, $\epsilon_{\mathrm{eff}}, \kappa$ and $b_{10} / b_{11}$, are given at half the plasma radius. The transport simulation results are obtained for 5 MW ECRH for both on- and off-axis deposition. 
significantly larger than for the X2-scenario of Sec. IV A with a vacuum $b_{01}=0.11$ (the same $n_{e}(r)$, but with strong off-axis power deposition). The equivalent transport simulation without the pronounced "electron-root" feature leads to smaller $I_{b}$ by a factor of about 2 . The impact of the toroidal mirror term is demonstrated by the scenarios $\mathbf{B}$ with $b_{01}=0.08$ and $\mathbf{C}$ with $b_{01}=0.12$ (in vacuum): $I_{b}$ is reduced with increasing $b_{01}$, but at the cost of degraded neoclassical confinement ( $\tau_{\mathrm{E}}^{\mathrm{n}}$ and $\langle\beta\rangle$ are much less affected than $\epsilon_{\mathrm{eff}}$ due to the strongly nonlinear temperature dependence of the neoclassical energy fluxes). For an increased $b_{01}$, the average $B_{0}$ must be reduced due to the ECRH resonance condition close to the "bean-shaped" plane (at the maximum of $B$ ) largely compensating the impact on $\langle\beta\rangle$ (compare also Sec. IV A for the high- and low- $t$ scenarios).

For the inward- (case D) and outward-shifted (case E) configurations with $i_{A}=-i_{B} \neq 0$ (see Sec. II A), the $b_{01}=0.1$ term is adjusted by varying the modular coil currents close to the "beanshaped" plane. With the same $b_{01}$ term, the inward and outward shift has only a small impact on $I_{b}$ and the neoclassical confinement both for on-axis and off-axis power deposition. Consequently, these shifts might also be used for a slight modification of the toroidal mirror term without changing the modular $i_{n}$.

The shape of the $B$-variation at the magnetic axis has a rather strong impact. In configuration $\mathbf{F}$, the toroidal range of the $B$ minimum is narrower, and broader for $\mathbf{G}$ with respect to the reference configuration A. Some freedom exists in the current distribution in the modular coils which can be used for an optimisation process based only on this coil system. For both cases $\mathbf{F}$ and $\mathbf{G}$, the narrower (broader) minimum $B$ range is obtained by increasing (decreasing) the current $i_{3}$, whilst keeping all other current ratios nearly the same. In the $b_{m n}$ spectrum, a second-harmonic term, $b_{02}=-0.021(\mathbf{F})$ and $b_{02}=0.023(\mathbf{G})$, is responsible for the width of the minimum $B$ range. With the additional $b_{02}$ term, the average magnetic field for $r \simeq 0$ is changed $\left(\left(b_{\max }-b_{\min }\right) / 2\right.$ is the toroidal ripple in this case). The neoclassical confinement characterised by $\epsilon_{\text {eff }}$ (for the $1 / \nu$ transport regime) is significantly affected whereas the configurational parameters $\kappa$ and $b_{10} / b_{11}$ are nearly unchanged. In the case $\mathbf{F}$ with the narrow minimum range, $I_{b}$ is significantly reduced, but the energy confinement is degraded, and vice versa for the broad minimum range $(\mathbf{G})$. The fairly strong difference in $\langle\beta\rangle$ is partly attributed to the average field (higher for $\mathbf{F}$ and lower for $\mathbf{G}$; this is different to the scenarios $\mathbf{B}$ and $\mathbf{C}$ ).

Next, the effect of higher $b_{m n}$ modes is analysed. In the case $\mathbf{H}$, only the $b_{01}, b_{10}$ and $b_{11}$ Fourier modes are taken into account for DKES and the transport calculations. The higher $b_{m n}$ modes have only a rather small effect on the neoclassical confinement reflected by $\tau_{\mathrm{E}}$ and $\langle\beta\rangle$, but have a strong 
impact on $I_{b}$. This result is in agreement with the simplified analysis of Ref. [17] (Fig. 6) based on mono-energetic transport coefficients only for one radius.

Finally, the magnetic configuration (case I) obtained from the "original" modular coil system as an outcome of the W7-X optimisation process (with identical currents in the modular coils leading to $b_{01}=0.10$ in vacuum; see Sec. I) is compared with case $\mathbf{A}$, i.e. the (standard) "high-mirror" W7-X configuration. The ratio $b_{10} / b_{11}$ is increased with respect to case $\mathbf{A}$ resulting in a reduced radial transport, but increased bootstrap current coefficients; comp. Fig. 4 in [17]. Again, this "old" analysis is confirmed by the transport simulations for both power depositions: $\tau_{\mathrm{E}}$ and $\langle\beta\rangle$ are increased, but $I_{b}$ is significantly larger. In this sense, the weight for minimised bootstrap current in the optimisation process is increased strongly for the existing W7-X coil system.

The configuration modifications $\mathbf{B}$ to $\mathbf{G}$ in the near vicinity of the (standard) "high-mirror" configuration $\mathbf{A}$ at $t_{a}=5 / 5$ demonstrate that the minimisation of $I_{b}$ is in conflict with neoclassical confinement improvement reflected by increased $\tau_{\mathrm{E}}^{\mathrm{n}}$ and $\langle\beta\rangle$. Transport simulations with "artificial" quasi-isodynamic corrections in the $b_{m n}$ spectrum, i.e. where the $b_{1-1}, b_{20}$ and both $b_{21}=b_{2-1}$ terms in the case $\mathbf{A}$ spectrum are replaced by the constraints given in [22], leads to significantly improved confinement but with large $I_{b}>0$. Consequently, quasi-isodynamicity mainly for deeply trapped particles is not sufficient to obtain high $\tau_{\mathrm{E}}^{\mathrm{n}}$ and $\langle\beta\rangle$ at low $I_{b}$ (the QIPC configuration analysed in [15] is very different to W7-X configurations). A variation of the $b_{10} / b_{11}$ ratio seems to be more efficient, but this ratio is nearly fixed by the W7-X coil system for a specific $t_{a}$ required for the island divertor.

A main part of this neccessary compromise between neoclassical confinement optimisation and minimisation of the bootstrap current is also reflected in the $t_{a}$-dependence; compare Sec. IV A. Here, the configuration parameters for the low- $t_{a}$ and high- $t_{a}$ cases with $b_{01} \simeq 0.1$ are briefly given. For $t_{a}=5 / 6$ with $\epsilon_{\text {eff }}=1.2 \%, \kappa=0.49$ and $b_{10} / b_{11}=0.51$ (at half the plasma radius), a rather large $I_{b}>0$ is obtained, whereas for $t_{a}=5 / 4$ with $\epsilon_{\mathrm{eff}}=3.1 \%, \kappa=0.38$ and $b_{10} / b_{11}=0.42$, even a fairly negative $I_{b}$ is found. Consequently, configurations with very different mirror terms $b_{01}$ are required for $I_{b} \simeq 0$ in the different $t_{a}$ scenarios.

\section{SUMMARY AND CONCLUSIONS}

Different quasi-stationary discharge scenarios have been analysed. For W7-X configurations with small or intermediate toroidal mirror terms, $b_{01}$, the predicted bootstrap current, $I_{b}$, is rather 
large and can only be balanced for high-power ECRH with ECCD in X2-mode at low or intermediate densities. An exception is the high $t_{a}=5 / 4$ configuration with a vacuum $b_{01} \simeq 0.04$, which allows for high density operation (in O2-mode) without ECCD. For high-performance discharges with high $\langle\beta\rangle \geq 3 \%$, the density must exceed the X2 cut-off limit, but the ECCD efficiency in O2-mode is rather low and thus insufficient to balance $I_{b}$. Consequently, "conservative" scenarios where the bootstrap current is controlled by the magnetic configuration are most promising. Except for the high- $t$ cases, only high-mirror configurations with $b_{01} \simeq 0.11$ for $t_{a}=5 / 5$ and with $b_{01} \simeq 0.25$ for $t_{a}=5 / 6$ are suited for scenarios with sufficiently small $I_{b}$.

Nevertheless, transport simulations with strong ECCD and $I_{b}+I_{\mathrm{ECCD}} \simeq 0$ have been performed in which $I_{\mathrm{ECCD}}<0$ reduces significantly the $t(r)$ at the radial ECCD position (increasing the bootstrap current coefficients in this region). These scenarios are not qualified for quasi-stationary discharges with high-performance (i.e. high density). Furthermore, low-order rationals appear in the $t$-profile both with positve and negative shear, $t^{\prime}$, which might lead to MHD activity.

For all the W7-X configurations under investigation, the minimisation of $I_{b}$ is in conflict with the neoclassical confinement improvement. Within any optimisation process, qualified target functions (here, e.g., $I_{b}, \tau_{\mathrm{E}}$ and $\langle\beta\rangle$ obtained from neoclassical transport simulations) together with the corresponding weights need to be defined (previous optimisations have been based on simple figures of merit, e.g. $\epsilon_{\mathrm{eff}}$ and the collisionless limit of the mono-energetic bootstrap current coefficent [40]). Additional constraints, like the proper operation of the island divertor in W7-X (this was not included in the "original" optimisation process), affect the weights of the target functions: the minimisation of $I_{b}$ in the magnetic configuration space becomes the highest priority and a (moderate) degradation of the neoclassical confinement must be accepted. Furthermore, the role of $\epsilon_{\text {eff }}$ (characterising only the $1 / \nu$ transport) is overestimated in comparison to $\tau_{E}$ and $\langle\beta\rangle$ (with smaller configurational dependence), where also the full nonlinear dependence on the simulated plasma parameters is taken into account. The ansatz of purely neoclassical transport modelling in most of the plasma volume leads to an upper limit for the confinement. Assuming strong turbulence would reduce the achivable $\tau_{\mathrm{E}}$ and $\langle\beta\rangle$.

In the next step of the discharge scenario development, the particle balance for calculating a selfconsistent density profile must be included in the iteration loop of equilibrium (VMEC code), neoclassical transport coefficients (DKES code), and self-consistent transport calculations (NTSS code). This extension, however, is not trivial: the particle sources mainly by recycling and pellet injection must be self-consistently calculated. Furthermore, a coupling to the edge plasma simulations 
(EMC3/EIRENE code) is essential.

\section{Acknowledgments}

The authors acknowledge very helpful discussions with P. Helander, C. Nührenberg and J. Nührenberg. This project has received funding from the European Union's Horizon 2020 research and inovation programme under grant agreement number 633053. The views and opinions expressed herein do not necessarily reflect those of the European Commission.

[1] G. Grieger et al., Phys. Fluids B 4, 2081 (1992).

[2] W. Lotz, J. Nührenberg, and C. Schwab, 13th International Conference Plasma Physics Controlled Nuclear Fusion Research, Washington, 1990 (IAEA, Vienna, 1991), Vol. 2, p. 603 ( http://wwwnaweb.iaea.org/napc/physics/FEC/STIPUP844_VOL2.pdf )

[3] J. Nührenberg and R. Zille, Phys. Lett. A 114, 129 (1986).

[4] C.D. Beidler and H. Maaßberg, Theory of Fusion Plasmas (Varenna 1996), Editrice Compositori, Bologna (1996), p. 375.

[5] H. Renner et al., Nucl. Fusion 40, 1083 (2000).

[6] H. Maßßberg, C.D. Beidler, and E.E. Simmet, Plasma Phys. Control. Fusion 41, 1135 (1999).

[7] S.P. Hirshman, W.I. van Rij, and P. Merkel, Comp. Phys. Comm. 43, 143 (1986).

[8] W.I. van Rij and S.P. Hirshman, Phys. Fluids B 1, 563 (1989).

[9] Y. Turkin et al., Phys. Plasmas 18, 022505 (2011).

[10] M. Yokoyama et al., Fusion Sci. Techn. 50, 327 (2006).

[11] M. Hirsch, J. Baldzuhn, and C.D. Beidler et al., Plasma Phys. Control. Fusion 50, 053001 (2008).

[12] A. Dinklage et al., Nucl. Fusion 53, 063022 (2013).

[13] A. Dinklage et al., Nucl. Fusion 47, 1265 (2007).

[14] H. Yamada et al., Nucl. Fusion 45, 1684 (2005).

[15] C.D. Beidler et al., Nucl. Fusion 51, 076001 (2011).

[16] N.B. Marushchenko et al., EPJ Web of Conf. 32, 01004 (2012).

[17] H. Maaßberg, W. Lotz, and J. Nührenberg, Phys. Fluids B 5, 3728 (1993). 
[18] J. Nührenberg and R. Zille, Theory of Fusion Plasmas (Varenna 1987), Editrice Compositori, Bologna (1988), p. 3.

[19] C. Schwab, Theory of Fusion Plasmas (Varenna 1988), Editrice Compositori, Bologna (1989), p. 85.

[20] A. Sengupta, J. Geiger, and P.J. Mc Carthy, Plasma Phys. Control. Fusion 49, 649 (2007).

[21] S. Gori, W. Lotz, and J. Nührenberg, Theory of Fusion Plasmas (Varenna 1996), Editrice Compositori, Bologna (1996), p. 335.

[22] C.D. Beidler and H. Maaßberg, Plasma Phys. Control. Fusions 43, 1131 (2001).

[23] M. Drevlak, J. Geiger, P. Helander, and Yu. Turkin, Nucl. Fusion 54, 073002 (2014).

[24] H.E. Mynick, T.K. Chu, and A.H. Boozer, Phys. Rev. Lett. 48, 322 (1982).

[25] K.C. Shaing and S.A. Hokin, Phys. Fluids 26, 2136 (1983).

[26] P. Helander, C.D. Beidler, and T.M. Bird et al., Plasma Phys. Control. Fusion 54, 124009 (2012).

[27] D. Lortz and J. Nührenberg, Z. Naturforsch. 31a, 1277 (1976) (http://zfn.mpdl.mpg.de/data/Reihe_A/31/ZNA-1976-31a-1277.pdf).

[28] O. Kaneko et al., Phys. Plasmas 9, 2020 (2002).

[29] A. Wakasa and S. Murakami, Plasma Fusion Res. 3, S1030 (2008).

[30] H.E. Mynick, Phys. Fluids 28, 1139 (1985).

[31] H. Maaßberg, C.D. Beidler, and Y. Turkin, Phys. Plasmas 16, 072504 (2009).

[32] N.B. Marushchenko, Y. Turkin, and H. Maaßberg, Comp. Phys. Comm. 185, 165 (2014).

[33] H. Maaßberg, C.D. Beidler, and N.B. Marushchenko, Phys. Plasmas 19, 102501 (2012).

[34] D. Reiter, The EIRENE Code User Manual, http://www.eirene.de, (2005).

[35] Y. Feng at al., Contrib. Plasma Phys. 54, 426 (2014).

[36] Y. Feng at al., Plasma Phys. Control. Fusion 53, 024009 (2011).

[37] K. Tanaka, K. Kawahata, and T. Tokuzawa et al., Fusion Sci. Techn. 58, 70 (2010).

[38] B. Pégourié et al., Nucl. Fusion 47, 44 (2007).

[39] H. Maaßberg et al., Plasma Phys. Control. Fusion 47, 1137 (2005).

[40] K.C. Shaing and J.D. Callen, Phys. Fluids 26, 3315 (1983). 